\title{
A Practical Guide for the Diagnosis of Primary Enteric Nervous System Disorders
}

\author{
${ }^{*}$ M.G. Schäppi, ${ }^{\dagger}$ A. Staiano, ${ }^{\ddagger} P . J$. Milla, ${ }^{\ddagger} V . V$. Smith, ${ }^{\S} J . A$. Dias, ${ }^{\|}$R. Heuschkel, ${ }^{\top} S$. Husby, \\ ${ }^{\#}$ M.L. Mearin, ${ }^{* *}$ A. Papadopoulou, ${ }^{\dagger \dagger}$ F.M. Ruemmele, \\ ${ }^{\S}$ Yvan Vandenplas, and ${ }^{|| \mid} S$. Koletzko
}

\begin{abstract}
Objective: Primary gastrointestinal neuropathies are a heterogeneous group of enteric nervous system (ENS) disorders that continue to cause difficulties in diagnosis and histological interpretation. Recently, an international working group published guidelines for histological techniques and reporting, along with a classification of gastrointestinal neuromuscular pathology. The aim of this article was to review and summarize the key issues for pediatric gastroenterologists on the diagnostic workup of congenital ENS disorders. In addition, we provide further commentary on the continuing controversies in the field.

Results: Although the diagnostic criteria for Hirschsprung disease are well established, those for other forms of dysganglionosis remain ill-defined. Appropriate tissue sampling, handling, and expert interpretation are crucial to maximize diagnostic accuracy and reduce interobserver variability. The absence of validated age-related normal values for neuronal density, along with the lack of correlation between clinical and histological findings, result in significant diagnostic uncertainties while diagnosing quantitative aberrations such as hypoganglionosis or ultrashort Hirschsprung disease. Intestinal neuronal dysplasia remains a histological description of unclear significance.

Conclusions: The evaluation of cellular quantitative or qualitative abnormalities of the ENS for clinical diagnosis remains complex. Such analysis should be carried out in laboratories that have the necessary expertise and access to their own validated reference values.
\end{abstract}

Received August 9, 2013.

From the *Pediatric Center, Clinique des Grangettes, and Centre Médical Universitaire, Geneva, Switzerland, the †Department of Translational Medical Science, Section of Pediatrics, University of Naples "Federico II," Naples, Italy, the †Departments of Gastroenterology and Histopathology, UCL Institute of Child Health and Great Ormond Street Hospital, London, UK, the §Department of Paediatrics, Hospital S. João, Porto, Portugal, the $\|$ Department of Paediatric Gastroenterology, Addenbrookes Hospital, Cambridge, UK, the $\uparrow$ Hans Christian Andersen Children's Hospital, OUH, Odense, Denmark, the \#Department of Paediatrics, Leiden University Medical Center, Leiden, The Netherlands, the **First Department of Paediatrics, University of Athens, Children's Hospital "Agia Sofia," Athens, Greece, §§UZ Brussel, Vrije Universiteit, Brussel, Brussels, Belgium, and the |||| Dr vonHaunersches Kinderspital, Ludwig-Maximilians-University, Munich, Germany.

Address correspondence and reprint requests to Prof Annamaria Staiano, Department of Translational Medical Science, Section of Pediatrics, University of Naples “Federico II," Naples, Italy (e-mail: staiano@ unina.it).

Drs Schäppi, Staiano, and Koletzko participated equally in this study.

Drs Milla and Smith served as guest authors as experts on enteric nervous disorders.

The authors report no conflicts of interest.

Copyright (C) 2013 by European Society for Pediatric Gastroenterology, Hepatology, and Nutrition and North American Society for Pediatric Gastroenterology, Hepatology, and Nutrition

DOI: 10.1097/MPG.0b013e3182a8bb50
Key Words: chronic intestinal pseudo-obstruction, gastrointestinal neuropathic disease, Hirschsprung disease, intestinal neuronal dysplasia, rectal suction biopsy

(JPGN 2013;57: 677-686)

ntestinal dysmotility disorders can be classified by etiology into primary visceral myopathies; primary visceral neuropathies; and secondary forms owing to toxic, metabolic, infectious, or other systemic disorders affecting the smooth muscle, interstitial cells of Cajal (ICC), or the enteric or extrinsic nervous systems. Symptoms of intestinal dysmotility, such as dyspepsia, vomiting, abdominal pain, bloating, diarrhea, and constipation, are nonspecific. The gastrointestinal (GI) transit may be too rapid or too slow or poorly coordinated. The most severe manifestation of dysmotility is chronic intestinal pseudoobstruction (CIPO) with signs and symptoms of bowel obstruction in the absence of a mechanical obstructive lesion. In general, there is a poor correlation among symptoms, the underlying etiopathology, and histopathological findings. For example, distinct phenotypes such a megacystis, microcolon, and hypoperistalsis syndrome may be caused by neuropathy or myopathy with different genetic traits (1).

Recently, an international working group (IWG) formed of pathologists, pediatric and adult gastroenterologists, and surgeons with special interest in neurogastroenterology-classified neuromuscular GI disorders based on the present evidence, the so-called London classification (2). In a previous article, this group had defined techniques for histopathological workup, interpretation, and reporting of intestinal neuromuscular disorders (3). A systematic review by the IWG revealed a lack of concordance in the quantitative assessment for elements of the enteric nervous system (ENS) (4).

The IWG acknowledged that most of the less well-understood entities have previously been described by their histomorphology, with little or no correlation to symptoms or natural history, and without comparison to any appropriate control group $(5,6)$. Only recently have the age of the patient and the location of biopsies been recognized as crucial to the interpretation of histopathological findings $(7,8)$. The clinical implications of many histopathological findings remain unclear, making it difficult for clinicians to provide families with any concrete idea of natural history of the disease.

The 3 recent articles $(2-4)$ by the IWG have tried to collate present knowledge in this field. Despite these comprehensive publications, the use of inaccurate terminology and vague diagnostic criteria remain common (9). In the authors' experience, inappropriate diagnoses such as hypoganglionosis and intestinal neuronal dysplasia (IND) are still being made based on symptoms and uncertain histomorphological findings, thereby perpetuating 
diagnostic confusion and uncertainty. In addition, inappropriate laboratory techniques and diagnostic criteria are still applied, leading to the prolonged misdiagnosis and potential of these children.

The purpose of this article was to extract and summarize the most important information from the IWG's 3 articles, placing them into a clinical context that is useful for practicing pediatric gastroenterologists. This medical position paper focuses only on congenital or developmental disorders of the ENS because these have proved particularly confusing. These disorders include more common diseases such as Hirschsprung disease (HD), monogenetic disorders with a clear clinical phenotype and well-defined histopathology, as well as ill-defined entities with no obvious etiology and little or no correlation among clinical phenotype, outcome, and histopathology. For clarity, we have not addressed acquired or secondary diseases of the ENS, for example, secondary to inflammation, degeneration of ganglion cells, or those characterized by abnormalities in glial or ICCs.

\section{ABSENCE OF NEURONS (AGANGLIONOSIS)}

HD

HD (or synonym Hirschsprung neurocristopathy) is characterized by the complete absence of enteric neurons in the myenteric and submucosal plexuses along a variable portion of the distal intestine. HD affects approximately 1 in 5000 newborns with a male:female ratio of approximately $4: 1$. HD, both sporadic and familial, has a complex genetic, sex-modified inheritance pattern and appears to be polygenic or multifactorial (10). Genetic factors have been identified in approximately $50 \%$ of patients with HD with a positive family history and in $15 \%$ to $20 \%$ of sporadic cases $(10,11)$. In almost all of the sporadic cases, RET proto-oncogene has been implicated, in part with other gene defects such as glial cell line-derived neurotrophic factor or the endothelin systems (10).

More than 10 genes mapping for transcription factors such as SOX10 and PHOX2B may be required for the HD phenotype to be fully expressed. HD occurs as an isolated trait in approximately $70 \%$ of cases (11). More frequent associated syndromes are Down syndrome, Waardenburg syndrome, and Smith-Lemli-Opitz syndrome (10). In a small number of cases $(<5 \%)$, the aganglionosis is a manifestation of multiple endocrine neoplasia (MEN) 2A, an autosomal dominant inherited disorder caused by certain germline mutations in the RET proto-oncogene with a high risk of medullary carcinoma of the thyroid and pheochromocytoma (12). In these cases, prophylactic thyroidectomy is recommended; however, routine screening for RET or other mutations in nonsyndromic forms is not yet practical and, because of the incomplete understanding of the complex genetic nature of the condition, not yet clinically indicated.

In the majority of cases $(80 \%)$, the aganglionic segment involves the rectum and the sigmoid colon only, whereas total colonic aganglionosis occurs in approximately $8 \%$ to $10 \%$ with an almost equal sex ratio. Rarely, aganglionosis extends into the small bowel or encompasses the entire bowel (subtotal or total intestinal aganglionosis) (Fig. 1) (10).

In these patients, the diagnosis of HD is particularly challenging because both histology (with the lack of increased acetyl cholinesterase staining) and barium enema (BE) (with the absence of a visible change in caliber of the colon) may be misleading.
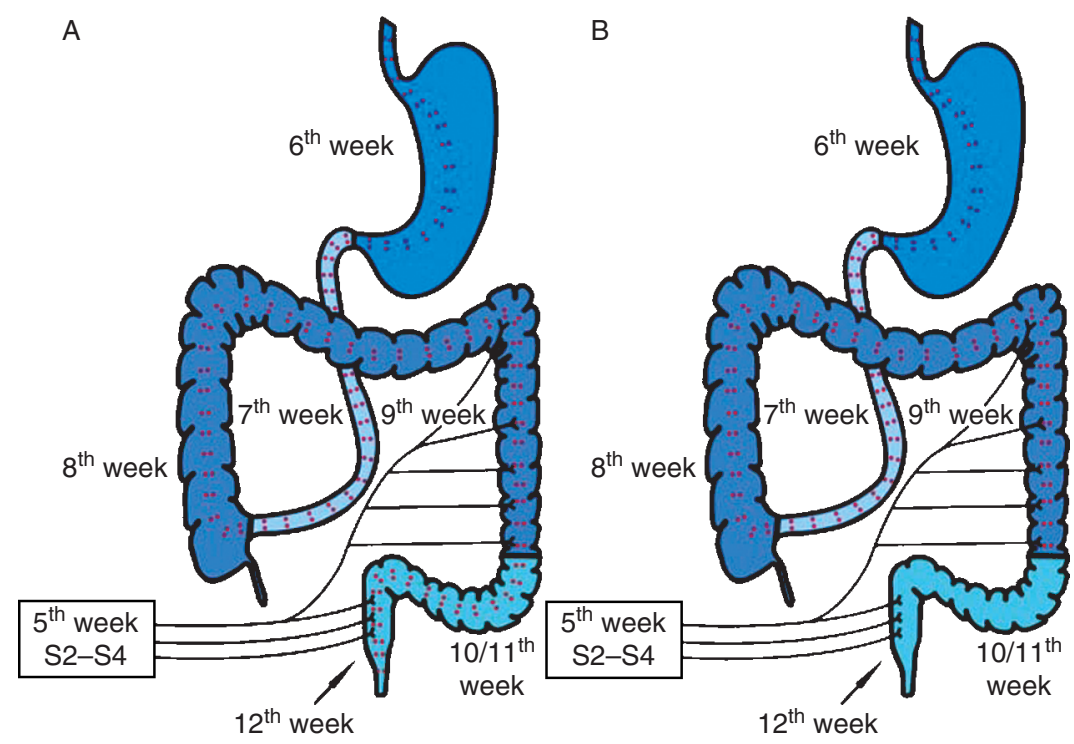

FIGURE 1. The intramural enteric nervous system develops from neural crest cells (NCCs) predominantly arising from the vagal neural crest. During the 7th week of gestational age, the first enteric NCCs are visible in the upper gastrointestinal tract and jejunum, and subsequently migrate distally to reach the rectum at week 12 (A). The submucosal plexus develops through migration of NCCs from the myenteric plexus, through the inner muscular layer into the submucosa. The extramural innervation consists of the sympathetic fibers from the ganglion mesentericum inferior. In addition, the distal colon (rectum, sigmoid, and a variable distance up to the splenic flexure) receives parasympathetic input from sacral nerves. In Hirschsprung disease (HD), the craniocaudal migration of NCCs is incomplete over a variable segment of the distal hindgut mostly in rectosigmoid (B). The acetylcholine (Ach) output remains unmodulated, and the high release of Ach induces increased acetycholinesterase (AchE) activity. The increased AchE activity is used as an additional diagnostic tool in short-segment HD if insufficient submucosa is available. The distribution of the parasympathetic extramural input explains why AchE staining is normal in aganglionic bowel proximal of the splenic flexure and in cases of total intestinal aganglionosis. 


\section{Clinical Presentation}

The clinical symptoms of HD usually start at birth with the delayed passage of meconium. More than $90 \%$ of term neonates, and $<10 \%$ of children with HD, pass meconium in the first 24 hours of life $(13,14)$. Thus, HD must be suspected in any full-term infant who does not pass meconium in the first 24 hours of life and in the premature infants who have excessively delayed the passage of meconium (7-8 days) (14). Failure of the distal bowel to relax and allow the passage of stool leads to functional obstruction and to secondary dilatation of the bowel proximal to the aganglionic segment. Affected children may present with severe dysmotility causing obstructive symptoms, ribbon-like stools, and frequently, failure to thrive. In $>90 \%$ of affected patients, the symptoms start during the neonatal period and in the majority, the diagnosis is made during the first 3 months of life, whereas $<1 \%$ are diagnosed during adult life (15). In these cases, constipation may be the only symptom. Enterocolitis and toxic megacolon with sudden onset of fever, abdominal distension, and explosive, at times bloody, diarrhea are the most feared complications of HD. Fecal stasis with proliferation of colonic bacteria may initiate enterocolitis (16) and therefore, a delayed diagnosis is thought to be a significant risk factor.

Surgery with resection of aganglionic bowel remains the criterion standard in the treatment of HD; however, despite adequate resection of the aganglionic segment, some patients may continue to have persistent bowel dysfunction. Postoperative bowel dysfunction includes constipation, fecal incontinence, and a continuous risk of enterocolitis. An altered distribution and impaired function of ICC, resulting in defective generation of electrical pacemaker activity, have been suggested to be one of the mechanisms contributing to dysmotility in patients operated on for HD (17).

\section{Current Diagnostic Protocol}

The diagnosis requires histopathological demonstration of the complete absence of enteric ganglion cells and presence of hypertrophied AchE-positive nerve trunks in the distal rectum thought to arise from the extrinsic innervation (2). Therefore, rectal biopsies with appropriate processing and interpretation by an experienced pathologist are the criterion standard to diagnose HD. Anorectal manometry (ARM) may be a confirmatory investigation or a tool to exclude HD in older children with a history of constipation from infancy. A BE should not be the first diagnostic investigation, but may be indicated in histologically confirmed cases to provide information on the length of the aganglionic bowel.

\section{Investigations}

\section{Histopathology}

Rectal Biopsies. The easiest means of obtaining adequate diagnostic tissue in rectal biopsies in infants is by rectal suction biopsy (RSB) (18). These provide larger and deeper biopsies than standard endoscopic forceps biopsies, which are usually inadequate to obtain sufficient submucosa and therefore are not recommended $(19,72)$ (Fig. 2). Compared with full-thickness rectal biopsies, RSB can be performed without general anesthesia or even sedation. The rates for major complications such as perforation, bleeding, and infection are between $1.3 \%$ and $2.9 \%(3,12,20)$. An accurate diagnosis is only possible if 2 to 3 suction biopsies are taken 2 to $3 \mathrm{~cm}$ above the dentate line and if they include sufficient submucosa (Fig. 2) (3). Biopsies taken closer to the dentate line may be misleading because of a normal zone of submucosal hypoganglionosis or even aganglionosis $(10 \mathrm{~mm}$ in neonates to $25 \mathrm{~mm}$ in children 3 years old and older (Fig. 3) $(3,18)$. If a full-thickness biopsy is obtained, this could be done as low as $1 \mathrm{~cm}$ above the dentate line because the myenteric plexus extends more distally. Between $9 \%$ and $30 \%$ of RSBs are inadequate and must be repeated, primarily because of insufficient submucosa in the biopsy $(21-23)$. This is particularly a problem in children older than 1 year. In this age group, rectal biopsies obtained with jumbo biopsy forceps (eg, Boston Scientific Radial Jaw 4, Boston Scientific, Natick, MA) may be an alternative (20) to a fullthickness biopsy (3).

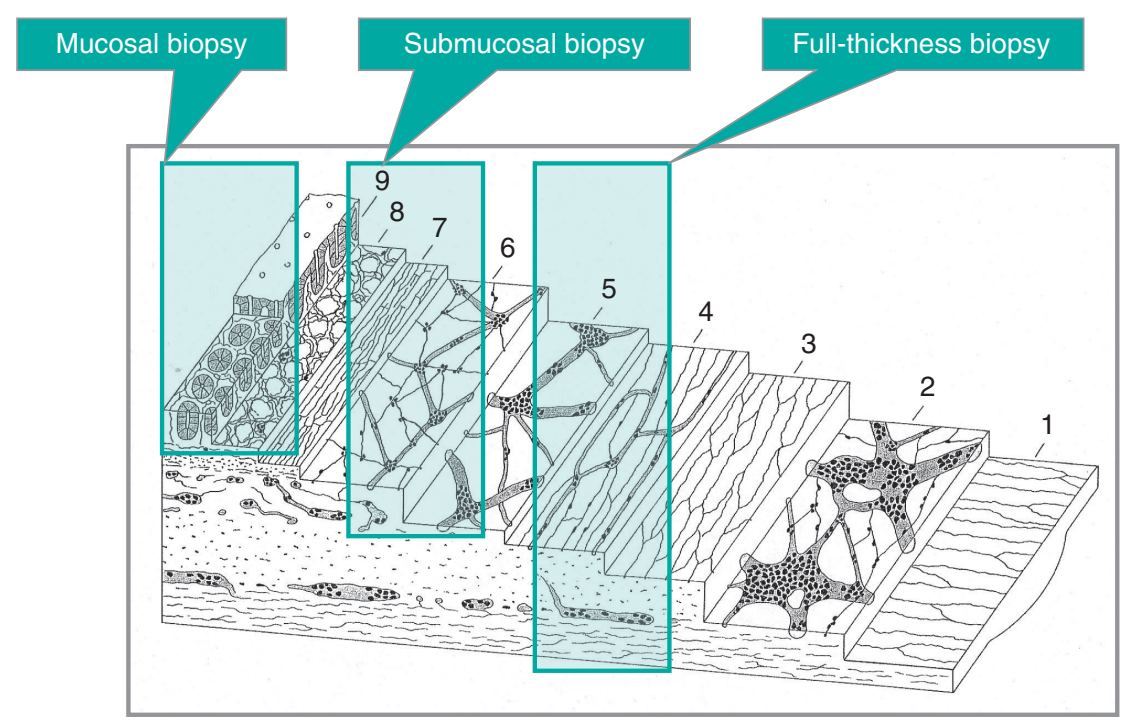

FIGURE 2. Different types of intestinal biopsies. A rectal suction biopsy allows a deeper biopsy, including the internal parts of the submucosal plexus compared with a mucosal biopsy taken during endoscopy. The myenteric plexus can only be viewed with a full-thickness biopsy. For an accurate diagnosis of Hirschsprung disease, especially proximal to the splenic flexure, a biopsy requires adequate submucosa to confirm the absence of ganglion cells. 1 =Plexus muscularis longitudinalis, $2=$ myenteric plexus, $3=$ plexus musculus circularis, $4=$ plexus submucosus extremus, 5 = plexus submucosus externus, $6=$ plexus submucosus internus, $7=$ plexus muscularis mucosae, $8=$ plexus mucosus subglandularis, $9=$ plexus mucosusperiglandularis. Modified after (72). 
A

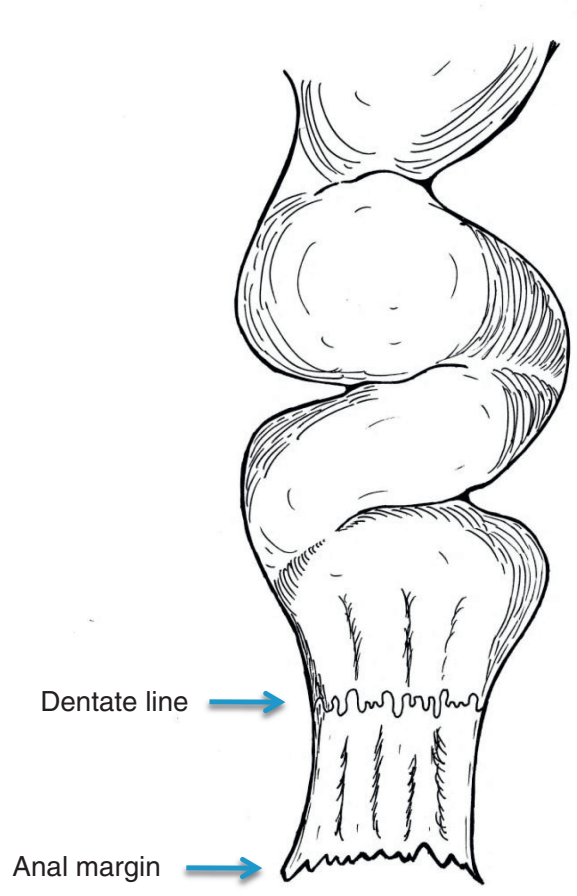

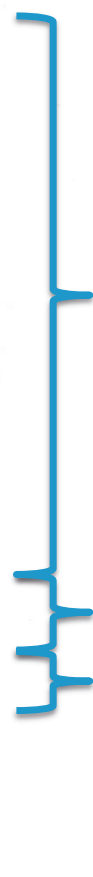

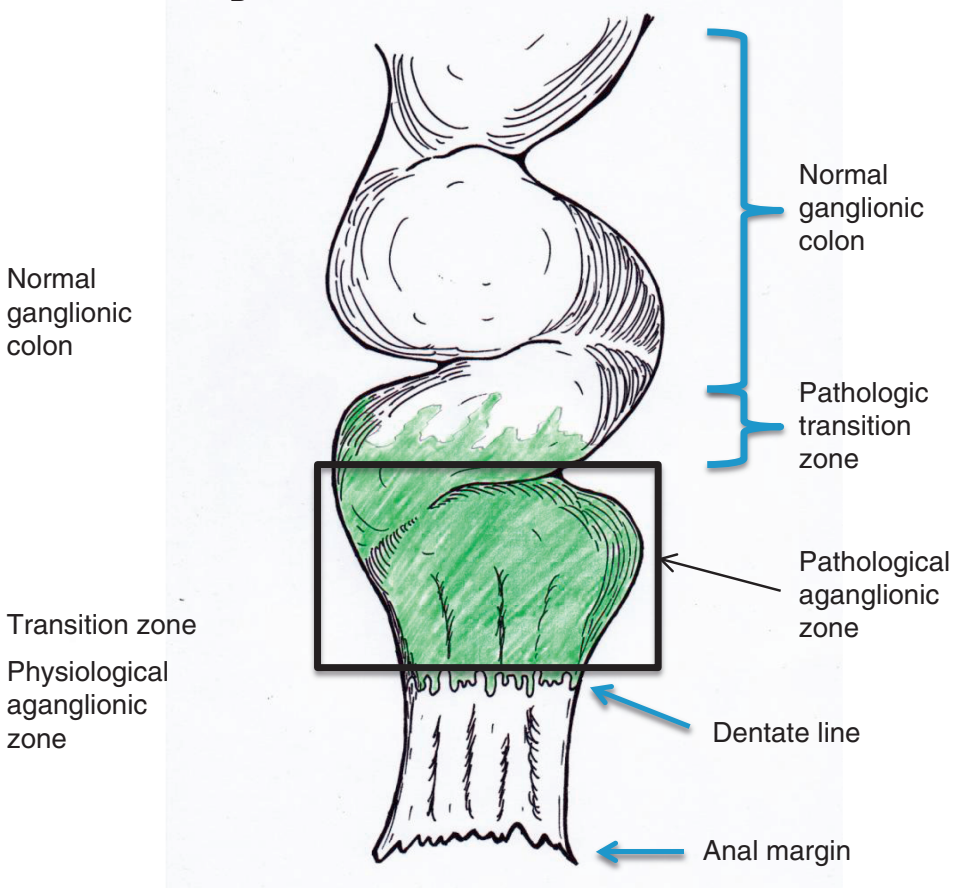

FIGURE 3. A, The normal rectum showing the physiological, hypoganglionic, or even aganglionic zone above the dentate line that transitions to the normal, more proximal, ganglionic bowel. Biopsies taken too close to the dentate line may be hypoganglionic or aganglionic and hence reported as false-positive for Hirschsprung disease. B, In Hirschsprung disease, the aganglionic zone extends from the dentate line proximally for a variable distance, before a transition zone leads to normal innervation. Modified after (18).

In conclusion, correctly performed RSBs taken 2 to $3 \mathrm{~cm}$ above the dentate line remain the preferred method in infants to obtain tissue for the diagnosis of HD (Fig. 3). The tissue must contain sufficient submucosa for the pathologist to confirm the absence of ganglion cells with certainty.

Staining of Biopsies. Once adequate biopsies are obtained, it is still controversial as to which histological methodology should be the preferred approach to confirm or exclude the diagnosis of HD. Techniques include analysis of hematoxylin and eosin (H\&E)stained paraffin sections, snap-frozen sections stained for acetyl cholinesterase enzyme histochemistry (AchE), and immunostaining for neuronal markers (3). All of the techniques have high sensitivity and specificity in an experienced laboratory with reported accuracy rates as high as $99 \%$, although the approach based entirely on histochemistry of frozen sections may be difficult in general pathology practices (5). In their consensus report, Knowles et al (3) concluded that insufficient data exist to firmly recommend one approach over the other, largely because of a lack of comparable studies comparing AchE histochemistry with enzyme histochemistry and immunostaining. American investigators favor the use of H\&E staining based on its availability in all centers, reliability, and low cost. The IWG of the London classification recommends an initial of 50 to $75 \mathrm{H} \& \mathrm{E}$-stained paraffin sections of each biopsy. The presence of a single unequivocal submucosal ganglion in RSB from the terminal $2 \mathrm{~cm}$ of rectum in H\&E-stained paraffin sections excludes the diagnosis of HD.

Although it is possible to establish a diagnosis of HD with the use of H\&E-stained paraffin sections alone, combining $\mathrm{H} \& \mathrm{E}$ staining with AchE enzyme histochemistry is still the most widely used method, particularly in Europe and Asia $(5,18)$. AchE staining allows the identification of thickened nerves in the muscularis mucosae in HD as early as 30 weeks' gestation and hypertrophic nerve trunks in the lamina propria of the mucosa, which are more common after 3 months of age (18). AchE staining alone may give false normal results in total colonic aganglionosis or in biopsies taken proximal to the splenic flexure because the activity of the extramural parasympathetic system from $\mathrm{S} 2$ to $\mathrm{S} 4$ decreases proximally from the anal canal, becoming almost absent at the splenic flexure (24-26) (Fig. 1). In these cases, diagnosis should not rely on mucosal and muscularis staining alone. The combination of AchE and H\&E staining on frozen sections is possible and increases the diagnostic accuracy because it improves the identification of submucosal ganglion cells, as well as demonstrates the abnormal nerve fiber distribution in the left colon (3).

Other enzyme histochemical techniques (all requiring frozen sections), such as lactate dehydrogenases (LDHs), may be used to identify neurons (27). The potential value of immunohistochemistry has been evaluated in a number of studies. Calretinin is an immunohistochemical marker that may be a potential alternative to AchE $(28-30)$. Calretinin is normally present in the perikarya and nerve processes of a subset of enteric ganglion cells. Immunoreactivity is lost in the aganglionic segment of $\operatorname{HD}(3,18)$. A recent comparative study shows that calretinin is as sensitive and specific as AchE and that the technique can be performed on paraffin sections (28); however, false-positive and false-negative results may occur with this technique $(29,31)$.

In conclusion, at the present time, either H\&E-stained paraffin-embedded sections or the analysis of frozen sections stained 
with H\&E plus AchE enzyme histochemistry should be used as the standard techniques to prove the absence of ganglion cells for the diagnosis of HD in rectal biopsies from children (3). AchE staining is not appropriate in long-segment $\mathrm{HD}$ and in biopsies proximal to the splenic flexure. If the success of the present studies on immunostaining is widely reproducible, it may be that H\&E along with immunostaining using markers such as calretinin will become the "new" standard for diagnosis.

\section{Intraoperative Histopathology}

Surgical resection of the aganglionic bowel and the hypoganglionic "transitional zone," which varies from a few millimetres to several centimetres $(32,33)$, is presently the only treatment for HD. At the time of resection, serial intraoperative frozen seromuscular biopsies should be obtained to map the aganglionic segment and to allow decisions to be made regarding the level of the proximal resection. Because the margin of transition from the hypoganglionic zone to normal innervation is not clearcut, the resection should be performed approximately 2 to $3 \mathrm{~cm}$ proximal to the first biopsy showing normal ganglion density. To confirm the intraoperative diagnosis, extemporaneous analyses of standard H\&E-stained sections or LDH enzyme histochemistry are sufficient (3). There should be good communication between the surgeon and the pathologist, and thicker tissue sections $(14-16 \mu \mathrm{m})$ should be used to avoid false-positive results for aganglionosis.

According to the London classification, the entire circumference of the proximal resection margin should be carefully evaluated to search for irregular distribution of ganglion cells in the transitional zone. Transverse sections should be taken at intervals needed to map the interface between aganglionic and ganglionic gut to within $1 \mathrm{~cm}$. One suggested approach is to sample the distal and proximal margins, the midpoint, and then more proximal midpoints between the last sample and the proximal margin until the distance is $<1 \mathrm{~cm}$. Another possibility is to obtain a longitudinal section through the entire length of resection (3). A reduction of ICC shown by c-kit labeling has been suggested in the aganglionic and the transition zone and even proximally in the normally innervated bowel (17). It has been postulated that this deficiency or disturbance of the networks of ICCs in the ganglionic bowel may cause the common dysmotility problems in children after surgery for HD; however, occurrence as a secondary phenomenon cannot be excluded. In summary, there is insufficient evidence to use c-kit staining for clinical decision making in the management of children with HD (34).

In conclusion, intraoperative biopsy sampling with adequate cutting and staining of the tissue is crucial to define aganglionic segments and should be available to children undergoing surgery for $\mathrm{HD}$.

\section{ARM}

ARM assesses the rectoanal inhibitory reflex (RAIR), which is absent in HD. Although the absence of the rectoanal inhibitory reflex is specific for the diagnosis of HD, the role of ARM is still debated. ARM has the advantages of being a less-invasive method without the exposure to ionizing radiation. The limitations include the need for the patient to be in a normal physiologic and quiet state to avoid possible artefacts $(35,36)$. A recent comprehensive systematic review by de Lorijn et al (35) compared the diagnostic accuracy among RSB, ARM, and BE for the diagnosis of HD. Although RSB gave the highest mean sensitivity and specificity (93\% and 98\%, respectively), ARM showed similar values $(91 \%$ and 94\%) (Table 1). Inconclusive results, however, are more common in ARM because of patient agitation (35). Because specificity is lower for ARM compared with RSB (36), ARM cannot reliably replace histology and biopsies.

ARM should not be used as a sole diagnostic tool for HD in neonates and infants; however; ARM is a useful screening test in older children presenting with chronic constipation and further symptoms suggesting HD (empty rectal ampulla, nonresponsiveness to standard therapy, early-onset constipation). If the rectoanal inhibitory reflex is absent, these patients should be referred for RSB to confirm the diagnosis of HD. If the rectoanal inhibitory reflex is present, HD could be reasonably excluded.

\section{$B E$}

Especially in young infants, a transitional zone is difficult to demonstrate (37). The technique also fails in children with total colonic aganglionosis, of whom $75 \%$ have a normal-caliber colon (37). Furthermore, BE may not distinguish HD from other newborn's pathologies, such as allergic colitis (38). A potential value of $\mathrm{BE}$ may be in helping to determine the length of an aganglionic segment. It may, however, not even be reliable in short-segment $\mathrm{HD}$; therefore, laparoscopic full-thickness or seromuscular biopsies are required to reliably map the aganglionic segment in longsegment HD $(39,40)$. If a $\mathrm{BE}$ is performed, this should be done without previous bowel preparation or recent digital rectal examination (41). In comparison with ARM and RSB, BE has a low sensitivity and specificity for the diagnosis of HD.

In conclusions, a BE should not be performed as an initial diagnostic tool because it does not represent a valid alternative to $\mathrm{RSB}$ or ARM to exclude or diagnose HD, regardless of age; however, BE may have some use as an additional investigation in diagnosed cases to assess the length of the rectosigmoid aganglionic segment before surgery.

\section{Ultrashort $H D$}

Ultrashort HD (UHD) is a controversial concept. Also described as achalasia of the internal anal sphincter (42), UHD has been reported with an incidence as high as $13.4 \%$ in a series of children diagnosed as having HD (43). In a retrospective study, Ciamarra et al (42) reported 20 cases of children with UHD. All of these children had severe constipation, absence of RAIR at the ARM, and normal rectal suction biopsy. Suggestive clinical symptoms included earlier onset of symptoms, lack of fecal soiling, and no history of withholding behavior (42). Histopathology cannot distinguish UHD from the physiologically aganglionic or hypoganglionic segment of the terminal rectum (44), given that in contrast to HD, no nerve fibers with increased AChE activity have been observed in the lamina propria mucosa in suspected UHD (43).

TABLE 1. Sensitivity and specificity of different diagnostic procedures for the diagnosis of HD

\begin{tabular}{lcc}
\hline & \multicolumn{2}{c}{ Parameter } \\
\cline { 2 - 3 } & $\begin{array}{c}\text { Mean } \\
\text { Index test }\end{array}$ & $\begin{array}{c}\text { Mean } \\
\text { specificity, \% } \\
(95 \% \text { CI })\end{array}$ \\
\hline Barium enema & $70(64-76)$ & $83(74-90)$ \\
Anorectal manometry & $91(85-95)$ & $94(89-97)$ \\
Rectal suction biopsy & $93(88-95)$ & $98(95-99)$ \\
\hline (stained for AchE activity) & & \\
\hline
\end{tabular}

Modified after (35). AchE $=$ acetycholinesterase; $\mathrm{CI}=$ confidence interval; $\mathrm{HD}=$ Hirschsprung disease. 
Indeed, proposed diagnostic criteria are the absence of the RAIR in a relaxed or sedated child in the presence of a normal biopsy; however, the diagnosis of UHD based only on the absence of the rectosphincteric reflex during ARM should cautiously be interpreted because the voluntary contraction of the external sphincter, which is common in anxious children with constipation, may mask the visibility of the internal sphincter relaxation on the tracing. Therapeutic options are represented by botulinum toxin injection or internal sphincter myectomy. Botulinum toxin injection has been reported as a less invasive and efficacious tool (42). The response to botulinum toxin injection may serve as both a diagnostic and therapeutic tool (42). If a decrease in anal sphincter pressure after the injection does not improve symptoms, it is unlikely that a myectomy would be curative.

In conclusion, because of the scarce published data, the existence of UHD in children must be further questioned; however, the absence of RAIR and a normal rectal biopsy, in the presence of typical symptoms, should suggest a diagnosis of UHD.

\section{QUANTITATIVE ABNORMALITIES OF NEURONAL DENSITY}

Although aganglionosis is relatively easy to diagnose as long as the tissue contains submucosa, the staining is correctly performed, and sufficient sections are viewed, the diagnosis of quantitative abnormalities such as hypoganglionosis or hyperganglionosis in the myenteric plexus remains extremely difficult. A prerequisite is an understanding of the normal neuronal density (4).

Values for neuronal density vary depending on the following:

1. Age of the child, with an inverse relation between nerves cells/ ganglia and age

2. Region of the examined bowel, fewer myenteric neurons in the small bowel than in the colon

3. Degree of intestinal dilatation, especially when the specimen is taken transversely along the long axis of the bowel

4. Type of biopsy and preparation (tissue sections vs whole-mount preparation)

5. Mode of staining (marker)

In their most recent publication, the IWG reviewed the 40 available studies on quantification of cellular components of the ENS in the normal human GI tract. The authors concluded that the lack of concordance between different investigators prevents the use of any robust values of the normal range of neuronal density (4). Most commonly, numbers of neurons are expressed per unit length (eg, per $10 \mathrm{~mm}$ ). The type of staining strongly determines the total number, with almost 30 -fold differences in values obtained with enzyme-histochemistry methods such as LDH, when compared with $\mathrm{H} \& \mathrm{E}$ staining. Application of antibodies that recognize neuronal antigens such as $\mathrm{HuC} / \mathrm{D}$ also obtains higher counts compared with $H \& E$ staining. Other factors that determine the quantitative assessment are section thickness, tissue size, the uneven circumferential distribution of ganglion cells and therefore the number of sections evaluated, and a high documented interobserver variation (32). To ensure the accurate estimates, particularly for hypoganglionosis, the average counts of at least 3 , and up to 5 , sections per staining at each level, of at least two-thirds of the circumference, are required $(4,32)$. Methodological recommendations for processing, staining, and counting for quantification of neuronal elements of the gut are provided by the IWG and should help to standardize the diagnostic workup in the future (4). With respect to the "ectopic" ganglia, the IWG recommends reserving this term for ganglion cells located within the muscularis propria (3). In contrast, ganglion cells in the lamina propria should not be considered a pathological finding in children, given that they have been found in the colon of healthy adults (3).

In conclusions, because of the methodological diversity and limitation of published data, no normal range of neuronal density can be provided for the different age groups and different regions of the bowel. Until the process is standardized, it is recommended that biopsies of patients with suspected quantitative abnormalities of cellular components of the ENS (hypoganglionosis and/or hyperganglionosis) should be evaluated by reference laboratories that have established their own control values, and are collected by the same observer with the use of standardized methods (4).

\section{Hypoganglionosis}

Intestinal hypoganglionosis is encountered either in association with $\mathrm{HD}$ or as an isolated condition. Hypoganglionosis occurs in the so-called transitional zone proximal to the aganglionic bowel in $\mathrm{HD}$, where it is more readily recognized. In contrast, isolated hypoganglionosis is an uncertain entity and present a diagnostic challenge. A recent systematic review identified only 92 published cases, of which 69 were boys and the average age at diagnosis was 4.8 years (45); however, the diagnosis of hypoganglionosis in these 92 cases remains extremely uncertain given the methodological problems of diagnostic workup used in these patients. Therefore, published data on clinical symptoms (ranging from slow transit constipation to enterocolitis and CIPO), imaging, manometric findings, and long-term outcome may not reflect true intestinal hypoganglionosis.

\section{Clinical Presentation}

Patients reported to have hypoganglionosis present with symptoms mimicking HD ranging from intractable slow transit constipation to CIPO requiring decompression surgery and parenteral nutrition. The outcome varies markedly and depends on the length of the affected bowel and the presence of complications, for example, from the use of parenteral nutrition. The overall reported mortality is $8 \%$ (45), mostly because of enterocolitis or complications of short bowel syndrome after recurrent surgical resections. In some patients, the dysmotility may also affect the urinary tract with megacystis, which may be visible on prenatal ultrasound. Recurrent urinary tract infections are a common problem and should be searched for actively.

\section{Investigations}

\section{Histopathology}

No validated lower limit of normal for the diagnosis of neuronal density is established, making the diagnosis of hypoganglionosis impossible based on published data alone (4). Therefore, the tissue should be assessed in a reference center with their own established normative values in children $(4,32)$.

Good liaison among the surgeon, the pediatric gastroenterologist, and the pathologist in the reference laboratory is essential for the correct handling, conservation, and transportation of the sample (3). The diagnosis can only safely be made on larger fullthickness surgical specimens of at least $1 \mathrm{~cm}$ in length covering at least two-thirds of the circumference (32). This means that rectal, seromuscular, or strips of full-thickness biopsies, are not sufficient to ascertain the diagnosis of hypoganglionosis (Fig. 2). Although no clear established guidelines exist, the IWG recommended in generalized disease that tissue should be taken from the first loop of jejunum, about $15 \mathrm{~cm}$ from the ligament of Treitz, and for localized disease from the most dilated area of bowel. No recommendation 
was made for children, but in our opinion, a full-thickness diagnostic sample in congenital ENS disorders should only be taken if decompression surgery of the gut is needed. Neuronal cell counts may be more accurate using immunohistochemistry for neuronal markers (eg, protein gene product 9.5, neuron-specific enolase, $\mathrm{HuC} / \mathrm{C}$ ) compared with $\mathrm{H} \& \mathrm{E}-$ stained slides (3); however, no study so far has addressed whether immunohistochemistry is superior to conventional staining for diagnosing decreased neuronal density (3). The methods used depend on the experience of the reference laboratory, but should take into consideration the published recommendations (3).

Conservative cutoff values for the diagnosis of hypoganglionosis in adults are suggested to be $<1$ ganglia per $10 \mathrm{~mm}$ with a mean number of $<2$ neurons per ganglion (3). For infants, particularly neonates, the lower limit may differ greatly (3). Neuronal counts in normal bowel in childhood have been published. The reported mean neuronal densities, made both on transverse sections (TS) and longitudinal sections (LS) to avoid the above-mentioned bias, were for jejunum 3.6 to $3.7 / \mathrm{mm}$ for TS-LS, respectively; for ileum 4.3/mm (TS, LS); and for colon 7 to $7.7 / \mathrm{mm}$ (TS-LS) (46). The literature shows discrepancies in the mean number of ganglion cells per centimetre, ranging from 5 to 149 (46-53). Because this variability exists in patients with normal innervation, it is clear that robust pathological abnormalities can be only determined once appropriated controls are established.

\section{Radiology}

Imaging and transit studies are helpful to rule out mechanical obstruction, but they are unlikely to provide a definitive diagnosis of the underlying condition (54).

\section{Manometry}

Manometry is recommended in patients with intractable chronic constipation who have failed medical management to exclude HD or in the absence of RAIR to prompt referral for rectal biopsy (55). Anorectal, antroduodenal, and colonic manometry can show a pattern of visceral neuropathy, with disturbed contractile activity, yet cannot identify the specific underlying pathophysiology from the observed motor pattern alone.

In conclusion, given the numerous factors that may cause small reductions in neuronal density and the difficulty in controlling for them, the IWG advised that diagnosis of mild or moderate hypoganglionosis based on quantification should be avoided at the present time. Thus, hypoganglionosis is a histological diagnosis, which should only be made by an expert pathologist, preferably on circumferential bowel sections of at least $1 \mathrm{~cm}$ in length. Findings should then include widely spaced myenteric ganglia that are small and contain grossly reduced numbers of neurons $(2,3)$.

\section{Ganglioneuromatosis in MEN 2B}

Transmural intestinal ganglioneuromatosis is a hallmark of MEN type 2B and associated with a gain in function mutation of the RET gene (54). Medullary thyroid carcinoma (MTC) develops extremely early in life, mostly in infancy, and affects all patients, whereas adrenal pheochromocytomas only affect $40 \%$ to $50 \%$ of patients with MEN 2B.

\section{Clinical Picture}

In most patients, disorders of GI motility are the first manifestations of the disease, but the presentation is variable both in severity and age of onset. Some patients present in early infancy, mimicking HD (56), and others develop the first GI symptoms in adult life (12). There is nearly always some colonic dysfunction and the most usual presentations are flatulence with abdominal distension, failure to thrive, chronic constipation, episodes of functional obstruction or Hirschsprung-like symptoms, diarrhea, and vomiting (12). The abnormality of the enteric nervous system is present along the entire GI tract, and the hyperplastic neural tissue (ganglioneuromata) may be seen by visual inspection of the mouth and lips, anal canal, or at GI endoscopy (57). They are associated with facial and musculoskeletal abnormalities such as pescavum, pectus excavatum, marfanoid features, and scoliosis (58). Occasionally, older children will present with either medullary carcinoma of the thyroid or with hypertension associated with a pheochromocytoma.

\section{Diagnosis}

Diagnosis requires either biopsy of obvious neuromata or rectal suction or full-thickness biopsy to demonstrate the characteristic histopathological changes. If genetic testing confirms MEN $2 \mathrm{~B}$, a prophylactic total thyroidectomy must be performed at that time, irrespective of age. Clumps of malignant cells in situ within the thyroid gland or an overt tumor have been detected within the first 2 months of life (56). Catecholamine and metabolite measurements provide the only means of screening for development of a pheochromocytoma.

\section{Investigations}

\section{Genetics}

The autosomal dominant pattern is almost always secondary to a specific activating mutation (Met918Thr in exon 16) in the RET proto-oncogene (59). This mutation often arises de novo and is associated with the most aggressive clinical form of MTC in children. Genetic testing is recommended in MEN 2 and should always form part of the management. An algorithm of a multistep process has recently been published (57).

\section{Biopsy and Histopathology}

As for HD, a biopsy requires adequate submucosa to demonstrate the characteristic findings of an increased density of nerve fibres and possibly ganglion cells in the submucosal and myenteric plexuses, with penetration of the hyperplastic nerve fibres into mucosa. The hyperplastic nerve fibers are accompanied by large ganglionic nodes containing numerous glial cells with an increased quantity of neurons. The changes are readily seen in H\&E-stained sections. AchE activity is not increased in the muscularis mucosae.

In conclusion, MEN 2B should be considered in infants presenting with HD-like symptoms or older children with features of the syndrome. Intestinal ganglioneuromatosis is a pathognomonic feature and can be identified on rectal suction or full-thickness biopsies. If genetic testing confirms the diagnosis, then thyroidectomy needs to be performed for early-onset MTC.

\section{IND}

The term neuronal intestinal dysplasia, first used by Nezelof et al (60) to describe hyperplasia of the myenteric plexus, renamed later by Meier-Ruge as IND (or IND type B) (61), is now considered as a morphologic phenotype affecting the submucosal plexus of the intestine, either in an isolated form or with known neuropathies such as HD or neurofibromatosis. Rather frequent changes in histological diagnostic criteria published during the last 4 decades have caused not only confusion but also scepticism about the existence of this condition as a discrete entity in both children and adults (62-65). Despite $>>250$ published articles on the subject, neither the 
diagnostic criteria nor is the clinical significance of the findings are agreed on. The latest morphometric criteria are summarized as follows: $>8$ neurons/ganglion (so-called giant ganglia) in $>20 \%$ of a minimum of 25 submucosal ganglia in patients older than 1 year (2). These criteria were developed with $15-\mu \mathrm{m}$-thick frozen sections and enzyme histochemistry. The correlation with counts on paraffin-embedded sections stained by H\&E or IHC is unclear (4).

\section{Controversies}

Some studies have challenged the dogma of giant ganglia as a diagnostic criterion: Lumb and Moore (66) have shown in an adult study the presence of up to $62 \%$ of ganglia with $>7$ neurons, making them a common feature. This clearly raises concerns that previous series included an unrepresentative cohort. Coerdt et al (7) have shown that in premature infants, the percentage of giant ( $>7$ cells) ganglia is up to $32.7 \%$, decreasing with increasing age: $21.5 \%$ in the first year and $16.5 \%$ in the 1 - to 14 -year-old group. Therefore, Koletzko et al (19) concluded that the criterion of hypercellularity is not sufficient to define IND as an independent morphological entity, but rather describes a developmental variation. This is also supported by the strong inverse correlation of "abnormal" morphologic features with age. Koletzko et al (19) found that biopsies from younger children were more often classified as compatible with IND or abnormal than those from older patients. In addition, interobserver agreement among the 3 experienced pathologists was not significantly different from that by chance, whereas all of the cases of HD were identified with $100 \%$ agreement (19). Highdensity submucosal ganglia with increased numbers of neurons were again found to be related to age in a blind assessment of rectal biopsies because these were present in $73 \%(22 / 30)$ of specimens taken in infants younger than 4 weeks, decreasing to $29 \%$ in older patients (67). A frequent history of prematurity has been noted in patients whose biopsies were reported to have changes initially consistent with IND but normalizing over time (68). Further evidence reinforces the relation of the morphologic findings of IND with age and immaturity, rather than suggesting this as a novel pathology $(47,69)$. The clinical significance also remains unclear because of the lack of correlation between clinical symptoms and histological findings (19). Clinical improvement with conservative treatment and spontaneous resolution is consistently reported (68-70), again arguing in favor of a developmental phenomenon or variation of normal rather than a pathological entity. A review of the literature supports this view and concludes that these appearances should not lead to major surgical intervention (67).

Morphologic features of IND have been reported in 2 siblings from a consanguine Turkish family with congenital short bowel syndrome and malrotation (71). This phenotype was described decades before and a monogenetic disorder had been suspected. Most recently, mutations in the Coxsackie- and adenovirus receptor-like membrane protein, which is essential for intestinal development and is important for junctional adhesion, have been described to be responsible for the disease; however, only 2 of 5 patients with Coxsackie- and adenovirus receptor-like membrane protein mutations causing short bowel syndrome revealed findings described as IND, and it is not clear whether the morphological features of IND disappeared with age; however, it cannot be excluded that in some children, this morphology may be a hind for a developmental disorder.

In conclusions, most of the evidence suggests that the histological appearance of so-called IND is a normal variant related to age. Owing to the lack of sufficient normative data, IND remains a histological description with poorly established clinical significance $(3,65)$.

\section{FUTURE DIRECTIONS}

Primary enteric neuropathies are for the most part rare but highly disabling diseases with a poor prognosis and a paucity of available therapeutic options. Unfortunately, much remains unanswered in understanding the pathogenesis of the majority of them. Nevertheless, this article presented an opportunity to better clarify the diagnostic approach of these patients, underlying the subtle differences among them, which makes difficult in most of the cases an accurate differential diagnosis. Further research needs to be focused on the development of comparable animal models as well as on larger clinical studies to solve many of the controversies. Continued exploration of the functional and morphological consequences of targeted gene mutations in animal models has the potential to increase the understanding of the causes of congenital disorders of GI motility. At the same time, it is essential to standardize uniform methods to characterize specific defects in tissue samples from patients with well-characterized GI motility. It is hoped that the London classification will enhance our ability to classify patients and provide better clinical and prognostic information. Improvement of this knowledge will promote the development of more targeted therapies to bridge the gap between basic science and clinical observation.

\section{SUMMARY AND RECOMMENDATIONS}

1. HD is a defined entity with clear diagnostic criteria.

a. Delayed passage of meconium and early-onset constipation are the closest clinical correlates.

b. Histology of adequate submucosal or full-thickness biopsies remain the mainstay for diagnosis and must include an absence of ganglion cells and, depending on biopsy site, hypertrophied nerve trunks.

c. Suction biopsies taken 2 to $3 \mathrm{~cm}$ above the dentate line remain the preferred method.

d. Either H\&E-stained paraffin-embedded sections or the analysis of frozen sections stained with $\mathrm{H} \& \mathrm{E}$ plus AchE enzyme histochemistry should be used as standard techniques for the diagnosis of HD in children.

e. Intraoperative biopsy sampling with adequate cutting and staining of the tissue is crucial to define aganglionic segments and surgical margins. This should be available to all of the children undergoing surgery for HD.

f. BE has no role in diagnosis but may be of value preoperatively to help determine the length of aganglionosis.

g. ARM has a role in excluding the diagnosis but only in older children with intractable constipation.

h. The absence of rectoanal inhibitory reflex and a normal rectal biopsy, in the presence of typical symptoms, should suggest a diagnosis of UHD.

2. Quantitative or qualitative neuronal abnormalities are less well defined and provide challenges for diagnosis.

a. Normative data of the ENS for the different age groups in pediatrics are urgently needed.

b. Radiological investigations and manometry may be helpful in the diagnostic workup of congenital dysmotility disorders, but cannot provide a definitive diagnosis.

c. Ganglioneuromatosis as a sign of MEN 2B can be established on appropriate specimens of rectal biopsies. Clear diagnosis, including genetic testing, needs to be made as early as possible to perform thyroidectomy before a medullary carcinoma develops.

d. Hypoganglionosis is an entity of uncertainty because the diagnosis is hampered by the lack of concordance for 
normative data among different investigators. Full-thickness, circumferential sections of at least $1 \mathrm{~cm}$ in length are required and should be examined in reference laboratories that have established their own normative age-related data.

e. IND is a morphological description with no correlation to clinical symptoms or outcome and therefore should not be used for clinical decision making.

Acknowledgments: The authors thank Prof Dr A.M. Muller, Bonn, Germany, and Prof Dr H.J. Krammer, Mannheim, Germany, for drawing Figures 1 and 2, respectively, and Prof Charles Knowles, Surgical Research Institute, Queen Mary University of London; Prof Dr Niels Quist, Pediatric Surgeon, Odense, Denmark; and Prof Dr Udo Rolle, Pediatric Surgeon, University of Frankfurt, Germany, for critically reviewing the manuscript.

\section{REFERENCES}

1. Gosemann J, Puri P. Megacystis microcolon intestinal hypoperistalsis syndrome: systematic review of outcome. Pediatr Surg Int 2011;27: 1041-6.

2. Knowles CH, De Giorgio R, Kapur RP, et al. The London Classification of gastrointestinal neuromuscular pathology: report on behalf of the Gastro 2009 International Working Group. Gut 2010;59:882-7.

3. Knowles CH, De Giorgio R, Kapur RP, et al. Gastrointestinal neuromuscular pathology: guidelines for histological techniques and reporting on behalf of the Gastro 2009 International Working Group. Acta Neuropathol 2009;118:271-301.

4. Knowles CH, Veress B, Kapur RP, et al. Quantitation of cellular components of the enteric nervous system in the normal human gastrointestinal tract-report on behalf of the Gastro 2009 International Working Group. Neurogastroenterol Motil 2011;23:115-24.

5. Martucciello G, Pini Prato A, Puri P, et al. Controversies concerning diagnostic guidelines for anomalies of the enteric nervous system: a report from the fourth International Symposium on Hirschsprung's disease and related neurocristopathies. J Pediatr Surg 2005;40:152731.

6. Meier-Ruge W, Longo-Bauer C. Morphometric determination of the methodological criteria for the diagnosis of intestinal neuronal dysplasia (IND B). Pathol Res Pract 1997;193:465-9.

7. Coerdt W, Michel J-S, Rippin G, et al. Quantitative morphometric analysis of the submucous plexus in age-related control groups. Virchows Arch 2004;444:239-46.

8. Wester T, O'Briain DS, Puri P. Notable postnatal alterations in the myenteric plexus of normal human bowel. Gut 1999;44:666-74.

9. Montedonico S, Caceres P, Munoz N, et al. Histochemical staining for intestinal dysganglionosis: over 30 years experience with more than 1500 biopsies. Pediatr Surg Int 2011;27:479-86.

10. Amiel J, Sproat-Emison E, Garcia-Barcelo M, et al. Hirschsprung disease, associated syndromes and genetics: a review. J Med Genet 2008;45:1-14.

11. Brooks A, Oostra B, Hofstra R. Studying the genetics of Hirschsprung's disease: unraveling an oligogenic disorder. Clin Genet 2005;67:6-14.

12. Cohen MS, Phay JE, Albinson C, et al. Gastrointestinal manifestations of multiple endocrine neoplasia type 2. Ann Surg 2002;235:64854discussion 54-5.

13. Clark DA. Times of first void and first stool in 500 newborns. Pediatrics 1977;60:457-9.

14. Bekkali N, Hamers SL, Schipperus MR, et al. Duration of meconium passage in preterm and term infants. Arch Dis Child Fetal Neonatal Ed 2008;93:F376-9.

15. Barnes PR, Lennard-Jones JE, Hawley PR, et al. Hirschsprung's disease and idiopathic megacolon in adults and adolescents. Gut 1986;27:53441.

16. Murphy F, Puri P. New insights into the pathogenesis of Hirschsprung's associated enterocolitis. Pediatr Surg Int 2005;21:773-9.

17. Rolle U, Piotrowska A, Nemeth L, et al. Altered distribution of interstitial cells of Cajal in Hirschsprung?s disease. Arch Pathol Lab Med 2002;126:928-33.
18. Scudiere JR, Maitra A, Montgomery EA. Selected topics in the evaluation of pediatric gastrointestinal mucosal biopsies. Adv Anat Pathol 2009; 16:154-60.

19. Koletzko S, Jesch I, Faus-Kebetaler T, et al. Rectal biopsy for diagnosis of intestinal neuronal dysplasia in children: a prospective multicentre study on interobserver variation and clinical outcome. Gut 1999;44: $853-61$.

20. Hirsch B, Angelides A, Goods S, et al. Rectal biopsies obtained with jumbo biopsy forceps in the evaluation of Hirschsprung disease. J Pediatr Gastroenterol Nutr 2011;52:429-32.

21. Alizai NK, Batcup G, Dixon MF, et al. Rectal biopsy for Hirschsprung's disease: what is the optimum method? Pediatr Surg Int 1998;13:121-4.

22. Croffie JM, Davis MM, Faught PR, et al. At what age is a suction rectal biopsy less likely to provide adequate tissue for identification of ganglion cells? J Pediatr Gastroenterol Nutr 2007;44:198-202.

23. Hayes C, Kawatu D, Mangray S, et al. Rectal suction biopsy to exclude the diagnosis of Hirschsprung disease. J Pediatr Gastroenterol Nutr 2012;55:268-71.

24. Barr LC, Booth J, Filipe MI, et al. Clinical evaluation of the histochemical diagnosis of Hirschsprung's disease. Gut 1985;26:393-9.

25. Kurer MH, Lawson JO, Pambakian H. Suction biopsy in Hirschsprung's disease. Arch Dis Child 1986;61:83-4.

26. Moore SW, Johnson G. Acetylcholinesterase in Hirschsprung's disease. Pediatr Surg Int 2005;21:255-63.

27. Meier-Ruge WA, Bruder E. Pathology of chronic constipation in pediatric and adult coloproctology. Pathobiology 2005;72:1-102.

28. Kapur R, Reed R, Finn L, et al. Calretinin immunohistochemistry versus acetylcholinesterase histochemistry in the evaluation of suction rectal biopsies for Hirschsprung disease. Pediatr Dev Pathol 2009;12:6-15.

29. Guinard-Samuel V, Bonnard A, De Lagausie P, et al. Calretinin immunohistochemistry: a simple and efficient tool to diagnose Hirschsprung disease. Mod Pathol 2009;22:1379-84.

30. Barshack I, Fridman E, Goldberg I, et al. The loss of calretinin expression indicates aganglionosis in Hirschsprung's disease. J Clin Pathol 2004;57:712-6.

31. Holland SK, Ramalingam P, Podolsky RH, et al. Calretinin immunostaining as an adjunct in the diagnosis of Hirschsprung disease. Ann Diagn Pathol 2011;15:323-8.

32. Swaminathan M, Kapur RP. Counting myenteric ganglion cells in histologic sections: an empirical approach. Hum Pathol 2010;41: 1097-108.

33. Schulten D, Holschneider A, Meier-Ruge W. Proximal segment histology of resected bowel in Hirschsprung's disease predicts postoperative bowel function. Eur J Pediatr Surg 2000;10:378-81.

34. Rolle U, Piaseczna-Piotrowska A, Puri P. Interstitial cells of Cajal in the normal gut and in intestinal motility disorders of childhood. Pediatr Surg Int 2007;23:1139-52.

35. de Lorijn F, Kremer LC, Reitsma JB, et al. Diagnostic tests in Hirschsprung disease: a systematic review. J Pediatr Gastroenterol Nutr 2006;42:496-505.

36. Jarvi K, Koivusalo A, Rintala RJ, et al. Anorectalmanometry with reference to operative rectal biopsy for the diagnosis/exclusion of Hirschprung's disease in children under 1 year of age. Int J Colorectal Dis 2009;24:451-4.

37. Taxman TL, Yulish BS, Rothstein FC. How useful is the barium enema in the diagnosis of infantile Hirschsprung's disease? Am J Dis Child $1986 ; 140: 881-4$

38. Bloom DA, Buonomo C, Fishman SJ, et al. Allergic colitis: a mimic of Hirschsprung disease. Pediatr Radiol 1999;29:37-41.

39. Mazziotti MV, Langer JC. Laparoscopic full-thickness intestinal biopsies in children. J Pediatr Gastroenterol Nutr 2001;33:54-7.

40. Carvalho JL, Soares-Oliveira M, Estevao-Costa J. Minimally invasive surgery on full-thickness intestinal biopsies. J Pediatr Gastroenterol Nutr 2002;34:240.

41. Reid JR, Buonomo C, Moreira C, et al. The barium enema in constipation: comparison with rectal manometry and biopsy to exclude Hirschsprung's disease after the neonatal period. Pediatr Radiol 2000;30:681-4.

42. Ciamarra $P$, Nurko $S$, Barksdale E, et al. Internal anal sphincter achalasia in children: clinical characteristics and treatment with clostridium botulinum toxin. J Pediatr Gastroenterol Nutr 2003;37:315-9. 
43. Meier-Ruge WA, Bruder E, Holschneider AM, et al. Diagnosis and therapy of ultrashort Hirschsprung's disease. Eur J Pediatr Surg 2004; 14:392-7.

44. Neilson IR, Yazbeck S. Ultrashort Hirschsprung's disease: myth or reality. J Pediatr Surg 1990;25:1135-8.

45. Dingemann J, Puri P. Isolated hypoganglionosis: systematic review of a rare intestinal innervation defect. Pediatr Surg Int 2010;26:1111-5.

46. Smith VV. Intestinal neuronal density in childhood: a baseline for the objective assessment of hypo- and hyperganglionosis. Pediatr Pathol 1993;13:225-37.

47. Aldridge RT, Campbell PE. Ganglion cell distribution in the normal rectum and anal canal. A basis for the diagnosis of Hirschsprung's disease by anorectal biopsy. J Pediatr Surg 1968;3:475-90.

48. Csendes A, Smok G, Braghetto I, et al. Histological studies of Auerbach's plexuses of the oesophagus, stomach, jejunum, and colon in patients with achalasia of the oesophagus: correlation with gastric acid secretion, presence of parietal cells and gastric emptying of solids. Gut 1992;33:150-4.

49. Krishnamurthy S, Heng Y, Schuffler MD. Chronic intestinal pseudoobstruction in infants and children caused by diverse abnormalities of the myenteric plexus. Gastroenterology 1993;104:1398-408.

50. Scharli AF, Sossai R. Hypoganglionosis. Semin Pediatr Surg 1998;7: 187-91.

51. Meier-Ruge WA, Brunner LA, Engert J, et al. A correlative morphometric and clinical investigation of hypoganglionosis of the colon in children. Eur J Pediatr Surg 1999;9:67-74.

52. Iwase H, Sadahiro S, Mukoyama S, et al. Morphology of myenteric plexuses in the human large intestine: comparison between large intestines with and without colonic diverticula. J Clin Gastroenterol 2005;39:674-8.

53. Coerdt W, Michel J-S, Rippin G, et al. Quantitative morphometric analysis of the submucous plexus in age-related control groups. Virchows Arch 2004;444:239-46.

54. Smith VV, Eng C, Milla PJ. Intestinal ganglioneuromatosis and multiple endocrine neoplasia type 2B: implications for treatment. Gut 1999; 45:143-6.

55. Dinning P, Benninga M, Southwell B, et al. Paediatric and adult colonic manometry: a tool to help unravel the pathophysiology of constipation. World J Gastroenterol 2010;16:5162-72.

56. Evans C, Nesbitt I, Walker J, et al. MEN 2B syndrome should be part of the working diagnosis of constipation of the newborn. Histopathology 2008;52:646-8.
57. Waguespack SG, Rich TA, Perrier ND, et al. Management of medullary thyroid carcinoma and MEN2 syndromes in childhood. Nature reviews. Endocrinology 2011;7:596-607.

58. Koletzko S, Schwarzer A. Other dysmotilies including chronic intestinal pseudo-obstruction syndrome. In: Kleinman R, Sanderson A, Goulet O, eds. Pediatric Gastrointestinal Disease. 5th ed Hamilton: BC Decker Inc; 2008:693-713.

59. Mulligan LM, Kwok JB, Healey CS, et al. Germ-line mutations of the RET proto-oncogene in multiple endocrine neoplasia type $2 \mathrm{~A}$. Nature 1993;363:458-60.

60. Nezelof C, Guy-Grand D, Thomine E. [Megacolon with hyperplasia of the myentericplexua. An anatomo-clinical entity, apropos of 3 cases]. Presse Med 1970;78:1501-6.

61. Meier-Ruge W. [Casuistic of colon disorder with symptoms of Hirschsprung's disease (author's trans1)]. Verh Dtsch Ges Pathol 1971;55:50610.

62. Berry CL. Intestinal neuronal dysplasia: does it exist or has it been invented? Virchows Arch A Pathol Anat Histopathol 1993;422:183-4.

63. Lumb PD, Moore L. Back to the drawing board. Intestinal neuronal dysplasia type B: not a histological entity yet. Virchows Arch 1998;432:99-102.

64. Kapur RP. Neuronal dysplasia: a controversial pathological correlate of intestinal pseudoobstruction. Am J Med Genet A 2003;122A:287-93.

65. de la Torre Mondragon L, Reyes-Mugica J. R.I.P. for IND B. Pediatr Dev Pathol 2006;9:425-6.

66. Lumb PD, Moore L. Are giant ganglia a reliable marker of intestinal neuronal dysplasia type B (IND B)? Virchows Arch 1998;432:103-6.

67. Cord-Udy CL, Smith VV, Ahmed S, et al. An evaluation of the role of suction rectal biopsy in the diagnosis of intestinal neuronal dysplasia. $J$ Pediatr Gastroenterol Nutr 1997;24:1-8.

68. Schofield DE, Yunis EJ. Intestinal neuronal dysplasia. J Pediatr Gastroenterol Nutr 1991;12:182-9.

69. Rintala R, Rapola J, Louhimo I. Neuronal intestinal dysplasia. Prog Pediatr Surg 1989;24:186-92.

70. Munakata K, Morita K, Okabe I, et al. Clinical and histologic studies of neuronal intestinal dysplasia. J Pediatr Surg 1985;20:231-5.

71. Van Der Werf CS, Wabbersen TD, Hsiao NH, et al. CLMP is required for intestinal development, and loss-of-function mutations cause congenital short-bowel syndrome. Gastroenterology 2012;142:453-62e3.

72. Wedel T, Roblick U, Gleiss J, et al. Organization of the enteric nervous system in the human colon demonstrated by wholemount immunohistochemistry with special reference to the submucous plexus. Ann Anat 1999;181:327-37. 